\title{
Diffractive parton distributions from the analysis with higher twist
}

\author{
Krzysztof Golec-Biernat ${ }^{(a, b) *}$ and Agnieszka $\operatorname{Luszczak}^{(b) \dagger}$ \\ ${ }^{a}$ Institute of Nuclear Physics Polish Academy of Sciences, Cracow, Poland \\ and \\ ${ }^{b}$ Institute of Physics, University of Rzeszów, Rzeszów, Poland
}

\begin{abstract}
Diffractive parton distributions of the proton are determined from fits to diffractive data from HERA. In addition to the twist-2 contribution, the twist-4 contribution from longitudinally polarised virtual photons is considered, which is important in the region of small diffractive masses. A new prediction for the longitudinal diffractive structure function is presented which differs significantly from that obtained in the pure twist- 2 analyses.
\end{abstract}

*e-mail: golec@ifj.edu.pl

†e-mail: agnieszka.luszczak@ifj.edu.pl 


\section{Introduction}

The diffractive deep inelastic scattering (DDIS) at HERA provide a very interesting example of the interplay between hard and soft aspects of QCD interactions. On one side, the virtuality of the photon probe is large $\left(Q^{2} \gg \Lambda_{Q C D}^{2}\right)$, while on the other side, the scattered proton remains almost intact, loosing only a small fraction of its initial momentum. Its transverse momentum with respect to the photon-proton collision axis is also small. In addition to the scattered incident particles, a diffractive system forms which is well separated in rapidity from the scattered proton. The most important observation made at HERA is that diffractive processes in DIS are not rare, quite the contrary, they constitute up to $15 \%$ of deep inelastic events. What's more, the ratio of the diffractive and inclusive cross sections is constant as a function of energy of the $\gamma^{*} p$ system or as a function of the photon virtuality. The latter fact reflects the logarithmic dependence on $Q^{2}$ of diffractive structure functions in the Bjorken limit.

In the $t$-channel picture, the diffractive interactions can be viewed as a vacuum quantum number exchange between the diffractive system and the proton. In old days of Regge phenomenology such a mechanism of interactions was termed a pomeron. With the advent of quantum chromodynamic we gain a new way of understanding the pomeron by modelling it with the help of gluon exchanges projected onto the color singlet state. In the lowest approximation, the pomeron is a two gluon exchange which is independent of energy. By considering radiative corrections to this process in the high energy limit, the famous BFKL pomeron [1-4] was constructed with a strong, power-like dependence on energy. This dependence ultimately violates unitarity which means that exchanges with more gluons have to be considered. A systematic program to sum exchanges with gluon number changing vertices was formulated in $[5,6]$ and developed in [7-10]. Other, somewhat more intuitive formulation, called Color Glass Condensate [11-14], is based on the idea of parton saturation [15] in which deep inelastic scattering occurs on a dense gluonic system in the proton. In these approaches unitarization is supposed to change the asymptotic energy behaviour of the cross sections involving the pomeron from power-like to logarithmic.

DDIS is particularly sensitive to the pomeron energy behaviour since diffractive scattering amplitudes are squared in diffractive cross sections. Thus, unitarization effects play more important role than for the total cross section which is proportional to the imaginary part of the scattering amplitude. This observation is a basis of a successful description of the first diffractive data from HERA in which the diffractive system was formed by the quark-antiquark $(q \bar{q})$ and quark-antiquark-gluon $(q \bar{q} g)$ systems. They can be viewed in the space of Fourier transformed transverse momenta as color dipoles $[16,17]$. In the approach we follow in the forthcoming presentation, the pomeron interaction is modelled by a two-gluon exchange which is subsequently substituted by the effective dipole-proton cross section fitted to inclusive DIS data $[18,19]$. In this way unitary is achieved.

In an alternative approach to DDIS, the diffractive structure functions are defined in terms of diffractive parton distributions (DPD). They are evolved in $Q^{2}$ with the help of the DokshitzerGribov-Lipatov-Altarelli-Parisi (DGLAP) equations [20-22]. Thus in the Bjorken limit, the diffractive structure functions depend logarithmically on $Q^{2}$, i.e. they provide the twist-2 description of DDIS. The theoretical justification of this approach is provided by the collinear factorisation theorem which is valid for hard diffractive scattering in ep collisions [23-27]. However, collinear factorisation fails in hadron-hadron scattering due to nonfactorizable soft interactions between incident hadrons $[28,29]$. Thus, unlike inclusive parton distributions, the DPD are not 


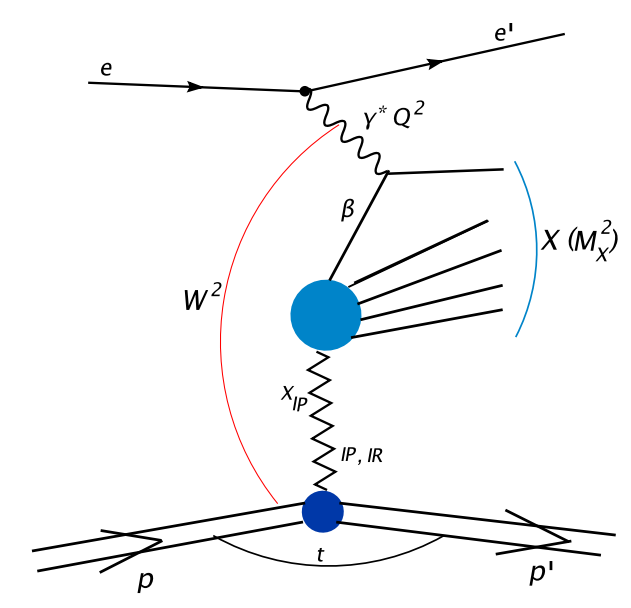

Figure 1: Kinematic variables relevant for diffractive DIS.

universal objects and in general can only be used for diffractive processes in the ep deep inelastic scattering. Nevertheless, the scale of nonuniversality can be estimated by applying them to hadronic reactions.

The relation between the color dipole approach with the $q \bar{q}$ and $q \bar{q} g$ diffractive components and the DGLAP based description was studied in detail in [30]. In short, after extracting the twist-2 part, the dipole approach provides $Q^{2}$-independent quark and gluon DPD. In addition, the $q \bar{q} g$ component, which was computed assuming strong ordering between transverse momenta of the gluon and the $q \bar{q}$ pair, gives the first step in the $Q^{2}$-evolution of the gluon distribution. The twist-2 approach, which is based on the DGLAP evolution equations, extends the two component dipole picture by taking into account more complicated diffractive final state. In the performed up till now twist-2 analyses, the diffractive parton distributions are determined through fits to diffractive data from HERA [31] We will follow this approach with an important modification.

The dipole approach teaches us one important lesson concerning the seemingly subleading twist -4 contribution, given by the $q \bar{q}$ pair from longitudinally polarised virtual photons $(L q \bar{q})$. Formally, it is is suppressed by a power of $1 / Q^{2}$ with respect to the leading twist -2 transverse contribution. However, the perturbative QCD calculation shows that for small diffractive masses, $M^{2} \ll Q^{2}$, the longitudinal contribution dominates over the twist-2 one which tends to zero in this limit. The effect of the $L q \bar{q}$ component is particularly important for the longitudinal diffractive structure function $F_{L}^{D}$ which is supposed to be determined from the high luminosity run data at HERA. Thus, we claim that it is absolutely necessary to consider the twist-4 contribution in the determination of the diffractive parton distributions. The analysis which we present confirms its relevance for the prediction for $F_{L}^{D}$, which differs significantly from that based on the pure DGLAP analysis. This is the main result of our paper.

The paper is organised as follows. In Section 2 we provide basic formulae for the kinematical variables and quantities measured in diffractive deep inelastic scattering. We also describe the three contributions which we include in the description of the diffractive structure functions, i.e. the twist-2, twist-4 and Regge contributions. In Section 3 we describe performed fits while in Section 4 we present their impact on the determination of the diffractive parton distributions and diffractive structure functions. We finish with conclusions and outlook. 


\section{Basic formulae}

We consider diffractive deep inelastic scattering: $e p \rightarrow e^{\prime} p^{\prime} X$, shown schematically in Fig. 1. After averaging over the azimuthal angle of the scattered proton, the four-fold differential cross section is given in terms of the diffractive structure functions $F_{2}^{D}$ and $F_{L}^{D}$ :

$$
\frac{d^{4} \sigma^{D}}{d \beta d Q^{2} d x_{\mathbb{P}} d t}=\frac{2 \pi \alpha_{e m}^{2}}{\beta Q^{4}}\left(1+(1-y)^{2}\right)\left\{F_{2}^{D}-\frac{y^{2}}{1+(1-y)^{2}} F_{L}^{D}\right\}
$$

where $y=Q^{2} /\left(x_{B} s\right)$ and $s$ is the $e p$ centre-of-mass energy squared. The expression in the curly bracket is called reduced cross section:

$$
\sigma_{r}^{D}=F_{2}^{D}-\frac{y^{2}}{1+(1-y)^{2}} F_{L}^{D} .
$$

Both structure functions depend on four kinematic variables $\left(\beta, Q^{2}, x_{\mathbb{P}}, t\right)$, defined as follows

$$
x_{\mathbb{P}}=\frac{Q^{2}+M^{2}-t}{Q^{2}+W^{2}}, \quad \beta=\frac{Q^{2}}{Q^{2}+M^{2}-t},
$$

where $-Q^{2}$ is virtuality of the photon, $t=\left(p-p^{\prime}\right)^{2}<0$ is the square of four-momentum transferred into the diffractive system, $M$ is invariant diffractive mass and $W$ is invariant energy of the $\gamma^{*} p$ system. The Bjorken variable $x_{B}=x_{\mathbb{P}} \beta$. For most of the diffractive events $|t|$ is much smaller then other scales, thus it can be neglected in eqs. (3). The diffractive structure functions are measured in a limited range of $t$, thus the integrated structure functions are defined

$$
F_{2, L}^{D(3)}\left(\beta, Q^{2}, x_{\mathbb{P}}\right)=\int_{t_{\min }}^{t_{\max }} d t F_{2, L}^{D}\left(\beta, Q^{2}, x_{\mathbb{P}}, t\right)
$$

The integrated reduced cross section $\sigma_{r}^{D(3)}$ is defined in a similar way.

\subsection{Twist-2 contribution}

In the QCD approach based on collinear factorisation, the diffractive structure functions are decomposed into the leading and higher twist contributions

$$
F_{2, L}^{D}=F_{2, L}^{D(t w 2)}+F_{2, L}^{D(t w 4)}+\cdots
$$

The twist-2 part is given in terms of the diffractive parton distributions through the standard collinear factorisation formulae [23,32-34]. In the next-to-leading logarithmic approximation we have

$$
\begin{aligned}
& F_{2}^{D(t w 2)}\left(x, Q^{2}, x_{\mathbb{P}}, t\right)=S_{D}+\frac{\alpha_{s}}{2 \pi}\left\{C_{2}^{S} \otimes S_{D}+C_{2}^{G} \otimes G_{D}\right\} \\
& F_{L}^{D(t w 2)}\left(x, Q^{2}, x_{\mathbb{P}}, t\right)=\frac{\alpha_{s}}{2 \pi}\left\{C_{L}^{S} \otimes S_{D}+C_{L}^{G} \otimes G_{D}\right\}
\end{aligned}
$$

where $\alpha_{s}$ is the strong coupling constant and $C_{2, L}^{S, G}$ are coefficients functions known from inclusive DIS $[35,36]$. The integral convolution is performed for the longitudinal momentum fraction

$$
(C \otimes F)(\beta)=\int_{\beta}^{1} d z C(\beta / z) F(z) .
$$


Notice that in the leading order, when terms proportional to $\alpha_{s}$ are neglected, the longitudinal structure function $F_{L}^{D(t w 2)}=0$. The functions $S_{D}$ and $G_{D}$ are given by diffractive quark and gluon distributions, $q_{D}^{f}$ and $g_{D}$ :

$$
\begin{aligned}
S_{D} & =\sum_{f=1}^{N_{f}} e_{f}^{2} \beta\left\{q_{D}^{f}\left(\beta, Q^{2}, x_{\mathbb{P}}, t\right)+\bar{q}_{D}^{f}\left(\beta, Q^{2}, x_{\mathbb{P}}, t\right)\right\} \\
G_{D} & =\beta g_{D}\left(\beta, Q^{2}, x_{\mathbb{P}}, t\right)
\end{aligned}
$$

Note that $\beta=x / x_{\mathbb{P}}$ plays the role of the Bjorken variable in DDIS. In the infinite momentum frame, the DPD are interpreted as conditional probabilities to find a parton with the momentum fraction $x=\beta x_{\mathbb{P}}$ in a proton under the condition that the incoming proton stays intact losing a small fraction $x_{\mathbb{P}}$ of its momentum. A formal definition of the diffractive parton distributions based on the quark and gluon twist-2 operators is given in [23, 25].

The DPD are evolved in $Q^{2}$ by the DGLAP evolution equations [37] for which the variables $\left(x_{\mathbb{P}}, t\right)$ play the role of external parameters. In this analysis we assume Regge factorisation for these variables:

$$
\begin{aligned}
& q_{D}^{f}\left(\beta, Q^{2}, x_{\mathbb{P}}, t\right)=f_{\mathbb{P}}\left(x_{\mathbb{P}}, t\right) q_{\mathbb{P}}^{f}\left(\beta, Q^{2}\right) \\
& g_{D}\left(\beta, Q^{2}, x_{\mathbb{P}}, t\right)=f_{\mathbb{P}}\left(x_{\mathbb{P}}, t\right) g_{\mathbb{P}}\left(\beta, Q^{2}\right) .
\end{aligned}
$$

For convenience, the functions $q_{\mathbb{P}}^{f}\left(\beta, Q^{2}\right)$ and $g_{\mathbb{P}}\left(\beta, Q^{2}\right)$ are called pomeron parton distributions. The motivation for such a factorisation is a model of diffractive interactions with a pomeron exchange [38]. In this model $f_{\mathbb{P}}$ is the pomeron flux

$$
f_{\mathbb{P}}\left(x_{\mathbb{P}}, t\right)=\frac{F_{\mathbb{P}}^{2}(t)}{8 \pi^{2}} x_{\mathbb{P}}^{1-2 \alpha_{\mathbb{P}}(t)},
$$

where $\alpha_{\mathbb{P}}(t)=\alpha_{\mathbb{P}}(0)+\alpha_{\mathbb{P}}^{\prime} t$ is the pomeron Regge trajectory and the formfactor

$$
F_{\mathbb{P}}^{2}(t)=F_{\mathbb{P}}^{2}(0) \mathrm{e}^{-B_{D}|t|}
$$

describes the pomeron coupling to the proton. We set $F_{\mathbb{P}}^{2}(0)=54.4 \mathrm{GeV}^{-2}[34], B_{D}=$ $5.5 \mathrm{GeV}^{-2}$ and $\alpha_{\mathbb{P}}^{\prime}=0.06 \mathrm{GeV}^{-2}$ [31], while the pomeron intercept $\alpha_{\mathbb{P}}(0)$ is fitted to data. The pomeron quark distributions are flavour independent, thus they are given by one function, a singlet quark distribution $\Sigma_{\mathbb{P}}$ :

$$
q_{\mathbb{P}}^{f}\left(\beta, Q^{2}\right)=\bar{q}_{\mathbb{P}}^{f}\left(\beta, Q^{2}\right) \equiv \frac{1}{2 N_{f}} \Sigma_{\mathbb{P}}\left(\beta, Q^{2}\right)
$$

where $N_{f}$ is a number of active flavours. The question about Regge factorisation is an issue which should be tested experimentally. In our approach, the pomeron is a model of diffractive

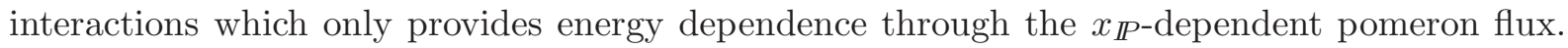
Its normalisation is only a useful convention since the normalisations of the pomeron parton distributions in eqs. (11) are fitted to data.

\subsection{Twist-2 charm contribution}

We describe the charm quark diffractive production using twist-2 formulae for the $c \bar{c}$ pair generation from a gluon. These are formulae analogous to the inclusive case in which the diffractive 

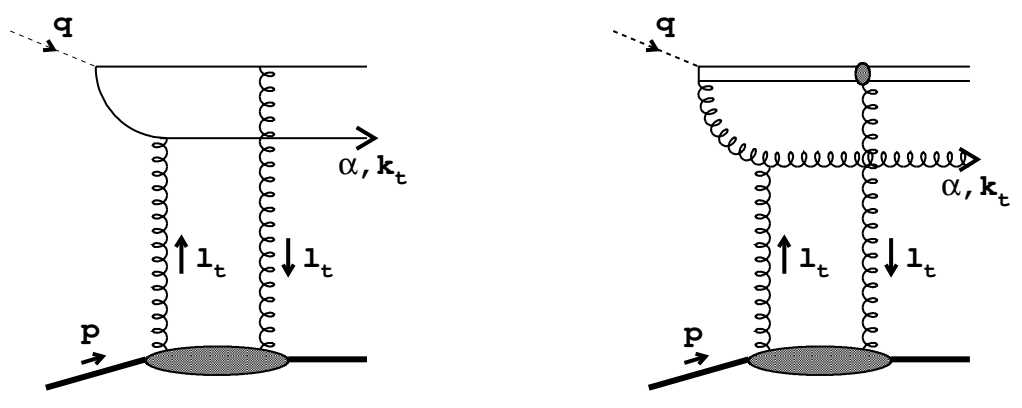

Figure 2: The $q \bar{q}$ and $q \bar{q} g$ components of the diffractive system in the dipole approach.

gluon distribution $g^{D}$ is substituted for the inclusive one [39]:

$$
F_{2, L}^{D(c \bar{c})}\left(\beta, Q^{2}, x_{\mathbb{P}}, t\right)=2 \beta e_{c}^{2} \frac{\alpha_{s}\left(\mu_{c}^{2}\right)}{2 \pi} \int_{a \beta}^{1} \frac{d z}{z} C_{2, L}\left(\frac{\beta}{z}, \frac{m_{c}^{2}}{Q^{2}}\right) g^{D}\left(z, \mu_{c}^{2}, x_{\mathbb{P}}, t\right)
$$

where $a=1+4 m_{c}^{2} / Q^{2}$ and the factorisation scale $\mu_{c}^{2}=4 m_{c}^{2}$ with the charm quark mass $m_{c}=1.4 \mathrm{GeV}$. The coefficient functions read

$$
\begin{gathered}
C_{2}(z, r)=\frac{1}{2}\left\{z^{2}+(1-z)^{2}+4 z(1-3 z) r-8 z^{2} r^{2}\right\} \ln \frac{1+\alpha}{1-\alpha} \\
+\frac{1}{2} \alpha\{-1+8 z(1-z)-4 z(1-z) r\} \\
C_{L}(z, r)=-4 z^{2} r \ln \frac{1+\alpha}{1-\alpha}+2 \alpha z(1-z)
\end{gathered}
$$

with $\alpha=\sqrt{1-4 r z /(1-z)}$. The $c \bar{c}$ pair can only be produced if invariant mass of the diffractive system $M^{2}$ fulfils the following condition

$$
M^{2}=Q^{2}\left(\frac{1}{\beta}-1\right)>4 m_{c}^{2} .
$$

\subsection{Twist -4 contribution}

The computation of the twist- 4 contribution, proportional to $1 / Q^{2}$, is a nontrivial task and one could be tempted to assume that this contribution is suppressed at large $Q^{2}$ as in inclusive DIS. However, by analysing diffractive final states in the dipole approach it was found that for diffractive mass $M^{2} \ll Q^{2}(\beta \rightarrow 1)$, the twist-4 contribution dominates over the vanishing twist-2 one $[19,40,41]$.

This observation is made on the basis of the perturbative QCD calculations in which the diffractive state is formed by the $q \bar{q}$ and $q \bar{q} g$ systems interacting with a proton through a colorless gluonic exchange which is a model of the pomeron interactions in QCD. In the simplest case, two gluons projected onto the color singlet state are exchanged, see Fig. 2. The computed amplitudes do not depend on energy in such a case which problem can be cured in a more sophisticated approach by modelling the dipole-proton cross section which fulfils unitarity conditions [19]. Independ of the details of the pomeron description, the diffractive mass (or $\beta$ ) dependence is a genuine prediction of pQCD calculations. It appears that the leading in $Q^{2}$ behaviour components, $q \bar{q}$ and $q \bar{q} g$ from transverse virtual photons, vanish for $\beta \rightarrow 1$. This is not the case for the $q \bar{q}$ production from longitudinal photons $(L q \bar{q})$ which is formally suppressed by $1 / Q^{2}$ 
with respect to the leading components. Thus, this particular $\beta$-dependence makes the $L q \bar{q}$ contribution dominant for $\beta \rightarrow 1$, see Fig. 3 .

The presence of the $L q \bar{q}$ component has important consequence for the longitudinal diffractive structure function which is supposed to be determined from the HERA data. The formula given below is an important element in the description of $F_{L}^{D}$ in the region of large $\beta$ :

$$
F_{L q \bar{q}}^{D}=\frac{3}{16 \pi^{4} x_{\mathbb{P}}} \mathrm{e}^{-B_{D}|t|} \sum_{f} e_{f}^{2} \frac{\beta^{3}}{(1-\beta)^{4}} \int_{0}^{\frac{Q^{2}(1-\beta)}{4 \beta}} d k^{2} \frac{k^{2} / Q^{2}}{\sqrt{1-\frac{4 \beta}{1-\beta} \frac{k^{2}}{Q^{2}}}} \phi_{0}^{2}\left(k, x_{\mathbb{P}}\right)
$$

where the function $\phi_{0}\left(k, x_{\mathbb{P}}\right)$ is given in terms of the dipole cross section $\hat{\sigma}\left(x_{\mathbb{P}}, r\right)$ and the Bessel functions $K_{0}$ and $J_{0}$ :

$$
\phi_{0}\left(k, x_{\mathbb{P}}\right)=k^{2} \int_{0}^{\infty} d r r K_{0}\left(\sqrt{\frac{\beta}{1-\beta}} k r\right) J_{0}(k r) \hat{\sigma}\left(x_{\mathbb{P}}, r\right) .
$$

Strictly speaking, eq. (20) contains all inverse powers of $Q^{2}$ but the part proportional to $1 / Q^{2}$ (called twist-4) dominates. The dipole-proton cross section describes the interaction of a color dipole, formed by the $q \bar{q}$ or $q \bar{q} g$ systems, with a proton. Following [18] we choose

$$
\hat{\sigma}\left(x_{\mathbb{P}}, r\right)=\sigma_{0}\left\{1-\exp \left(-r^{2} Q_{s}^{2} / 4\right)\right\}
$$

where $Q_{s}^{2}=\left(x_{\mathbb{P}} / x_{0}\right)^{-\lambda} \mathrm{GeV}^{2}$ is a saturation scale which provides the energy dependence of the twist -4 contribution. The parameters $\sigma_{0}=29 \mathrm{mb}, x_{0}=4 \cdot 10^{-5}$ and $\lambda=0.28$ are taken from [18] (Fit 2 with charm). This form of the dipole cross section provides successful description of the first HERA data on both inclusive and diffractive structure functions [18,19]. A different parametrisation of $\hat{\sigma}$, without the saturation scale, is also given in [42-44]. We checked that a very similar description of $F_{L q \bar{q}}^{D}$ was found in a recent analysis [45] based on the Color Glass Condensate parameterisation of the dipole scattering amplitude [46].

The relation between the dipole approach with three diffractive components and the DGLAP approach with diffractive parton distributions was analysed at length in [30]. Summarising this relation, the twist -2 part of the $q \bar{q}$ component gives a diffractive quark distribution. The twist-2 part of the $q \bar{q} g$ component forms a first step of the DGLAP evolution which starts from a given gluon distribution. Both diffractive parton distributions do not depend on $Q^{2}$, thus they may serve as initial conditions for the DGLAP equations at the scale which is not determined. From this perspective, the DGLAP approach offers a description of more complicated diffractive state with any number of partons ordered in transverse momenta. However, the pQCD calculations tell us that the twist- 2 analysis of diffractive data should include the twist -4 contribution since it cannot be neglected at large $\beta$. This is the strategy which we follow in our analysis.

We also borrow from the dipole approach a general form in $\beta$ of the initial quark distribution which vanishes at the endpoints $\beta=0,1$ (see eq. (31) in which $A_{q}$ and $C_{q}$ are positive). A very important aspect of Regge factorisation (11) can also be motivated by the dipole approach. It is a consequence of geometric scaling of the dipole cross section (22) [30,47].

\subsection{Reggeon contribution}

The diffractive data from the H1 collaboration for higher values of $x_{\mathbb{P}}$ hints towards a contribution which decreases with energy. This effect can be described by reggeon exchanges in addition 
to the rising with energy pomeron exchange. Following $[48,49]$, we consider the dominant isoscalar $\left(f_{2}, \omega\right)$ reggeon exchanges which lead to the following contribution to $F_{2}^{D}$ :

$$
F_{2}^{D(R)}=\sum_{R} f_{R}\left(x_{\mathbb{P}}, t\right) F_{R}\left(\beta, Q^{2}\right) .
$$

This contribution breaks Regge factorisation of the diffractive structure function, however, its presence is necessary for $x_{\mathbb{P}}>0.01$ [50]. The reggeon flux $f_{R}$ is given by the formula analogous to eq. (13)

$$
f_{R}\left(x_{\mathbb{P}}, t\right)=\frac{F_{R}^{2}(0)}{8 \pi^{2}} \mathrm{e}^{-|t| / \Lambda_{R}^{2}}\left|\eta_{R}(t)\right|^{2} x_{\mathbb{P}}^{1-2 \alpha_{R}(t)},
$$

where $\alpha_{R}(t)=0.5475+1 \cdot t$ is the reggeon trajectory. From the Regge phenomenology of hadronic reactions $\Lambda_{R}=0.65 \mathrm{GeV}$ and the reggeon-proton couplings are given by [49]: $F_{f_{2}}^{2}(0)=$ $194 \mathrm{GeV}^{-2}$ and $F_{\omega}^{2}(0)=52 \mathrm{GeV}^{-2}$. The functions

$$
\left|\eta_{R}(t)\right|^{2}=4 \cos ^{2}\left[\pi \alpha_{R}(t) / 2\right], \quad\left|\eta_{R}(t)\right|^{2}=4 \sin ^{2}\left[\pi \alpha_{R}(t) / 2\right]
$$

are signature factors for even $\left(f_{2}\right)$ and odd $(\omega)$ reggeons, respectively. We could also consider isovector reggeons $\left(a_{2}, \rho\right)$ but their couplings to the proton are much smaller and we neglect them. Finally, the reggeon structure function $F_{R}$ is given by [49]

$$
F_{R}(\beta)=A_{R} \beta^{-0.08}(1-\beta)^{2},
$$

where the normalisation $A_{R}$ is a fitted parameter. Thus, in the first approximation, we neglect the $Q^{2}$-dependence of the reggeon contribution.

\section{$3 \quad$ Fit details}

\begin{tabular}{|l||c|c|c|c|c|}
\hline Collab. & No. points & Data & $|t|$-range & $Q^{2}$-range & $\beta$-range \\
\hline \hline H1 $[50]$ & 72 & LP & {$[0.08,0.5]$} & {$[2,50]$} & {$[0.02,0.7]$} \\
\hline ZEUS [51] & 80 & LP & {$[0.075,0.35]$} & {$[2,100]$} & {$[0.007,0.48]$} \\
\hline H1 $[31]$ & 461 & $M_{Y}<1.6$ & {$\left[\left|t_{\min }\right|, 1\right]$} & {$[3.5,1600]$} & {$[0.01,0.9]$} \\
\hline ZEUS [52] & 198 & $M_{Y}<2.3$ & {$\left[\left|t_{\min }\right|, \infty\right]$} & {$[2.2,80]$} & {$[0.003,0.975]$} \\
\hline
\end{tabular}

Table 1: Kinematic regions of diffractive data from HERA. LP means leading proton data and $M_{Y}$ is invariant mass of a dissociated proton. Dimensionfull quantities are in units of $1 \mathrm{GeV}$.

In our analysis we use diffractive data from the H1 [31,50] and ZEUS $[51,52]$ collaborations. In Table 1 we show their kinematic limits in which they have been measured. The minimal value value of $|t|$ is given by

$$
\left|t_{\text {min }}\right| \simeq \frac{x_{\mathbb{P}}^{2}}{1-x_{\mathbb{P}}} m_{p}^{2}
$$

where $m_{p}$ is the proton mass. The leading proton data from $\mathrm{H} 1$, measured in the range given in Table 1, were corrected by the $\mathrm{H} 1$ collaboration to the range $\left|t_{\text {min }}\right|<|t|<1 \mathrm{GeV}^{2}$.

The ZEUS data are given for the diffractive structure function $F_{2}^{D}$, thus we use in our analysis the following formulae

$$
\begin{aligned}
& F_{2}^{D}=F_{2}^{D(t w 2)}+F_{2}^{D(R)}+F_{L q \bar{q}}^{D} \\
& F_{L}^{D}=F_{L}^{D(t w 2)}+F_{L q \bar{q}}^{D} .
\end{aligned}
$$




\begin{tabular}{|c||c||c||c|c||c|c|c||c|c|c||c|}
\hline No & Data & Fit & $\alpha_{\mathbb{P}}(0)$ & $A_{R}$ & $A_{q}$ & $B_{q}$ & $C_{q}$ & $A_{g}$ & $B_{g}$ & $C_{g}$ & $\chi^{2} / N$ \\
\hline \hline 1 & H1 (LP) & tw-2 & 1.098 & 0.29 & 1.75 & 1.49 & $0.5^{*}$ & 2.09 & 0.67 & 0.80 & 0.48 \\
\hline 2 & ZEUS (LP) & tw-2 & 1.145 & 1.05 & 2.13 & 1.51 & $0.5^{*}$ & $10.0^{*}$ & 1.03 & 2.26 & 0.40 \\
\hline \hline 3 & H1 & tw-2 & 1.117 & 0.49 & 1.33 & 1.63 & 0.34 & 0.17 & -0.16 & -1.10 & 1.04 \\
\hline 4 & & tw- $(2+4)$ & 1.119 & 0.48 & 1.62 & 1.98 & 0.59 & 0.04 & -0.56 & -1.68 & 1.17 \\
\hline \hline 5 & ZEUS & tw-2 & 1.093 & $0.0^{*}$ & 1.68 & 1.01 & $0.5^{*}$ & 0.49 & -0.03 & -0.40 & 1.35 \\
\hline 6 & & tw- $(2+4)$ & 1.092 & $0.0^{*}$ & 1.20 & 0.85 & 0.57 & 0.07 & -0.52 & -1.48 & 1.82 \\
\hline
\end{tabular}

Table 2: The fit parameters to H1 nd ZEUS data. The presence of twist-4 in the fits is marked by tw- $(2+4)$. The parameters with an asterisk are fixed in the fits.

The longitudinal twist-4 contribution is present on the r.h.s. of eq. (28) since $F_{2}^{D}$ is the sum of the contributions from the transverse and longitudinal polarised virtual photon. The H1 data, however, are presented for the reduced cross section (2). Thus we substitute relations (28) and (29) in there and use

$$
\sigma_{r}^{D}=\left\{F_{2}^{D(t w 2)}+F_{2}^{D(R)}-\frac{y^{2}}{1+(1-y)^{2}} F_{L}^{D(t w 2)}\right\}+\frac{2(1-y)}{1+(1-y)^{2}} F_{L q \bar{q}}^{D} .
$$

The expression in the curly brackets is the twist -2 contribution while the last term is the twist- 4 one. Notice that the difference between $F_{2}^{D}$ and $\sigma_{r}^{D}$ is most important for $y \rightarrow 1$.

We fit the diffractive parton distributions at the initial scale $Q_{0}^{2}=1.5 \mathrm{GeV}^{2}$, assuming the Regge factorised form (111) with the following pomeron parton distributions [31]:

$$
\begin{aligned}
& \beta \Sigma_{\mathbb{P}}(\beta)=A_{q} \beta^{B_{q}}(1-\beta)^{C_{q}} \\
& \beta g_{\mathbb{P}}(\beta)=A_{g} \beta^{B_{g}}(1-\beta)^{C_{g}} .
\end{aligned}
$$

The six indicated parameters are fitted to data. We additionally multiplied both distributions by a factor $\exp \{-a /(1-\beta)\}$ with $a=0.01$ to secure their vanishing for $\beta=1$. This factor is only important when $C_{q}$ or $C_{g}$ becomes negative in the fits. For the evolution, we use the next-to-leading order DGLAP equations with $\Lambda_{Q C D}=407 \mathrm{MeV}$ for $N_{f}=3$ flavours [53].

The pomeron flux in eq. (11) is integrated over $t$ in the limits given in Table 1 which leads to the form

$$
f_{\mathbb{P}}\left(x_{\mathbb{P}}\right)=\frac{F_{\mathbb{P}}^{2}(0)}{8 \pi^{2} B}\left\{\mathrm{e}^{-B\left|t_{\min }\right|}-\mathrm{e}^{-B\left|t_{\max }\right|}\right\} x_{\mathbb{P}}^{1-2 \alpha_{\mathbb{P}}(0)} .
$$

The shrinkage parameter $B$ equals

$$
B=B_{D}+2 \alpha_{\mathbb{P}}^{\prime} \ln \left(1 / x_{\mathbb{P}}\right)
$$

with $B_{D}=5.5 \mathrm{GeV}^{-2}$ and $\alpha_{\mathbb{P}}^{\prime}=0.06 \mathrm{GeV}^{-2}[50]$.

In summary, we have eight fit parameters altogether: the pomeron intercept $\alpha_{\mathbb{P}}(0)$, reggeon normalisation $A_{R}$ in eq. (26) and six parameters in eqs. (31132)

\section{Fit results}

The data sets from Table1 were obtained in different kinematical regions, using different methods of their analysis. Thus, we decided to perform fits to each data set separately. The values of the fit parameter are shown in Table 2. The difference between them can be attributed to the scale of uncertainty of our analysis. In each case we preformed two fits: with and without the twist -4 formula added to the twist- 2 contribution. 


\subsection{Leading proton data}

We started from fits to the leading proton data. The fit parameters in this case are displayed in the first two rows of Table 2. We only show the twist-2 fit results since they are not changed in fits with the twist -4 term. This happenes because the leading proton data comes from the region of $\beta$ values where the twist -4 contribution is small $(\beta \leq 0.7$ for $\mathrm{H} 1$ and $\beta<0.5$ for ZEUS), see Fig. 3 ,

The data with a dissociated proton (DP) which are measured in the region of large $\beta$ influence most the value of the parameter $C_{g}$ which controls the behaviour of the gluon distribution at $\beta \rightarrow 1$. For the LP data $C_{g}$ is positive and the gluon distribution is suppressed near $\beta \approx 1$, while for the DP data $C_{g}$ is negative and the gluon distribution is strongly enhanced. This shows that the data with $\beta>0.7$ are crucial for the proper analysis. Without this kinematic region we lose important information about diffractive interactions. Thus, from now on we concentrate on the analysis of the DP data.

\subsection{H1 data}

The fit parameters to the $\mathrm{H} 1$ data with a dissociate proton are given in the third and fourth rows of Table 2. We see that the fit quality is practically the same for both fits, with and without the twist-4 contribution. The presence of the reggeon term improves fit quality by 30 units of $\chi^{2}$ for 461 experimental points. A good quality of the fits is illustrated in Fig. 4 which also shows that the reduced cross sections (30) from the twist-2 (solid lines) and twist-(2+4) fits (dashed lines) are very close to each other.

In Fig. 5 we show our results on the reduced cross section for the largest measured value of $\beta=0.9$. In this region, the twist -4 contribution, shown by the dotted lines, cannot be neglected. We see that the curves from both the twist-2 (solid) and twist- $(2+4)$ (dashed) fits describe data reasonable well. However, the curves with twist-4 have a steeper dependence on $x_{\mathbb{P}}$ (energy) than in the pure twist-2 analysis. This observation is by far more pronounced in the analysis of the ZEUS data performed for the structure function $F_{2}^{D}$.

The diffractive parton distributions from our fits are shown in Fig. 6 in terms of the pomeron parton distributions, $\beta \Sigma_{\mathbb{P}}\left(\beta, Q^{2}\right)$ and $\beta g_{\mathbb{P}}\left(\beta, Q^{2}\right)$. Being independent of the pomeron flux, such a presentation allows for a direct comparison of the results from fits to different data sets. We see that the singlet quark distributions are quite similar while the gluon distributions are different. In the fit with twist-4, the gluon distribution is stronger peaked near $\beta \approx 1$. This somewhat surprising result can be understood by looking at the logarithmic slope of $F_{2}^{D}$ for fixed values of $\beta$. From the LO DGLAP equations we have schematically:

$$
\frac{\partial F_{2}^{D}}{\partial \ln Q^{2}} \sim \frac{\partial \Sigma_{\mathbb{P}}}{\partial \ln Q^{2}}=P_{q q} \otimes \Sigma_{\mathbb{P}}+P_{q G} \otimes G_{\mathbb{P}}-\Sigma_{\mathbb{P}} \int P_{q q}
$$

where the negative term describes virtual corrections. For large $\beta$, the measured slope is negative which means that the virtual emission term must dominate over the real emission ones. The addition of the twist-4 contribution to $F_{2}^{D}$, proportional to $1 / Q^{2}$, contributes a negative value to the slope which has to be compensated by a larger gluon distribution in order to describe the same data.

In Fig. 7 we present our most important results. On the left panel, the $F_{2}^{D}$ structure function is shown from both fits, with and without the twist- 4 contribution (shown by the dotted lines). 
We see no significant difference between these two results. However, the longitudinal structure function $F_{L}^{D}$ differs significantly for the two fits (right panel) due to the twist-4 contribution. Let us emphasise that both sets of curves were found in the fits which well describe the existing data on $\sigma_{r}^{D}$, including the large $\beta$ region. Thus, an independent measurement of $F_{L}^{D}$ in this region would be an important test of the QCD mechanism of diffraction.

\subsection{ZEUS data}

The results of same fits performed for the ZEUS data are shown in the last two rows of Table 2 , This time the Regge contribution (26) is not necessary since fits give the reggeon normalisation $A_{R} \approx 0$. In general, the fit quality is worse than for the $\mathrm{H} 1$ data.

As shown in Fig. 8, the biggest difference between the twist -2 and twist- $(2+4)$ results occurs at large $\beta$ values. This is analysed in detail in Fig. 9. We see that the presence of the twist-4 term in the fit (dashed lines) improves the agreement with the data in this region. In particular, a steep dependence of $F_{2}^{D}$ on $x_{\mathbb{P}}$ is better reproduced by the twist- $(2+4)$ fit then by the twist-2 one (solid lines). This dependence is to large extend driven by the twist-4 contribution (dotted lines).

The behaviour of the diffractive parton distributions and structure functions, shown in Figs. 10 and 11, respectively, is very similar to that found for the H1 data. The gluon distribution from the fit with twist- 4 is stronger peaked near $\beta \approx 1$ and the longitudinal structure functions in the large $\beta$ region is dominated by the twist -4 contribution.

We summarise the effect of the twist-4 contribution in Fig. 12 showing the predictions for the longitudinal diffractive structure function $F_{L}^{D}$. Ignoring this contribution, we find the two solid curves coming from the pure twist-2 analysis of the H1 (upper) and ZEUS (lower) data. With twist-4, the dashed curves are found, the upper one from the H1 data and the lower one from the ZEUS data. There is a significant difference between these two predictions in the region of large $\beta$. We believe that the effect of the twist- 4 contribution will be confirmed by the forthcoming analysis of the HERA data.

\section{Conclusions}

We performed fits of the diffractive parton distributions to new diffractive data from the $\mathrm{H} 1$ and ZEUS collaborations at HERA. In addition to the standard twist-2 formulae, we also considered the twist- 4 contribution which dominates in the region of large $\beta$. This contribution comes from the diffractive production of the $q \bar{q}$ pair by the longitudinally polarised virtual photons. The effect of the twist -4 contribution on the diffractive parton distributions and structure functions was carefully examined. The twist -4 contribution leads to the gluon distribution which is peaked stronger at $\beta \approx 1$ than in the case without twist-4.

The main result of our analysis is a new prediction for the longitudinal diffractive structure function $F_{L}^{D}$. The twist-4 term significantly enhances $F_{L}^{D}$ in the region of large $\beta$. A measurement of this function at HERA in the region of large $\beta$ should confirm the presented expectations which are based on the perturbative QCD calculations. The obtained diffractive parton distributions can also be used in the analysis of diffractive processes at the LHC, in particular, to the estimation of the background to the diffractive Higgs production, see [54] for a recent discussion. 


\section{Acknowledgements}

This research has been partly supported by MEiN research grant 1 P03B 02828 (2005-08) and by EU grant HEPTOOLS, MRTN-CT-2006-035505. The hospitality of the Galileo Galilei

Institute for Theoretical Physics in Florence and partial support of INFN during the completion of this work is gratefully acknowledged.

\section{References}

[1] V. S. Fadin, E. A. Kuraev and L. N. Lipatov, Phys. Lett. B60, 50 (1975).

[2] L. N. Lipatov, Sov. J. Nucl. Phys. 23, 338 (1976).

[3] E. A. Kuraev, L. N. Lipatov and V. S. Fadin, Sov. Phys. JETP 45, 199 (1977).

[4] I. I. Balitsky and L. N. Lipatov, Sov. J. Nucl. Phys. 28, 822 (1978).

[5] J. Bartels, Nucl. Phys. B151, 293 (1979).

[6] J. Bartels, Nucl. Phys. B175, 365 (1980).

[7] J. Bartels, Phys. Lett. B298, 204 (1993).

[8] J. Bartels, Z. Phys. C60, 471 (1993).

[9] J. Bartels and M. Wusthoff, Z. Phys. C66, 157 (1995).

[10] J. Bartels and C. Ewerz, JHEP 09, 026 (1999), [hep-ph/9908454].

[11] L. D. McLerran and R. Venugopalan, Phys. Rev. D49, 2233 (1994), [hep-ph/9309289].

[12] L. D. McLerran and R. Venugopalan, Phys. Rev. D49, 3352 (1994), [hep-ph/9311205].

[13] E. Iancu and R. Venugopalan, hep-ph/0303204.

[14] E. Iancu, hep-ph/0608086.

[15] L. V. Gribov, E. M. Levin and M. G. Ryskin, Phys. Rept. 100, 1 (1983).

[16] N. N. Nikolaev and B. G. Zakharov, Z. Phys. C49, 607 (1991).

[17] N. Nikolaev and B. G. Zakharov, Z. Phys. C53, 331 (1992).

[18] K. Golec-Biernat and M. Wusthoff, Phys. Rev. D59, 014017 (1999), [hep-ph/9807513].

[19] K. Golec-Biernat and M. Wusthoff, Phys. Rev. D60, 114023 (1999), [hep-ph/9903358].

[20] V. N. Gribov and L. N. Lipatov, Sov. J. Nucl. Phys. 15, 438 (1972).

[21] G. Altarelli and G. Parisi, Nucl. Phys. B126, 298 (1977).

[22] Y. L. Dokshitzer, Sov. Phys. JETP 46, 641 (1977). 
[23] A. Berera and D. E. Soper, Phys. Rev. D53, 6162 (1996), [hep-ph/9509239].

[24] J. C. Collins, Phys. Rev. D57, 3051 (1998), [hep-ph/9709499].

[25] F. Hautmann, Z. Kunszt and D. E. Soper, Phys. Rev. Lett. 81, 3333 (1998), [hep$\mathrm{ph} / 9806298]$.

[26] F. Hautmann, Z. Kunszt and D. E. Soper, Nucl. Phys. B563, 153 (1999), [hep-ph/9906284].

[27] J. Blumlein, B. Geyer and D. Robaschik, Nucl. Phys. B755, 112 (2006), [hep-ph/0605310].

[28] J. C. Collins, L. Frankfurt and M. Strikman, Phys. Lett. B307, 161 (1993), [hep$\mathrm{ph} / 9212212]$.

[29] M. Wusthoff and A. D. Martin, J. Phys. G25, R309 (1999), [hep-ph/9909362].

[30] K. Golec-Biernat and M. Wusthoff, Eur. Phys. J. C20, 313 (2001), [hep-ph/0102093].

[31] H1, A. Aktas et al., Eur. Phys. J. C48, 715 (2006), [hep-ex/0606004].

[32] L. Trentadue and G. Veneziano, Phys. Lett. B323, 201 (1994).

[33] A. Berera and D. E. Soper, Phys. Rev. D50, 4328 (1994), [hep-ph/9403276].

[34] J. C. Collins, J. Huston, J. Pumplin, H. Weerts and J. J. Whitmore, Phys. Rev. D51, 3182 (1995), [hep-ph/9406255].

[35] W. Furmanski and R. Petronzio, Phys. Lett. B97, 437 (1980).

[36] W. Furmanski and R. Petronzio, Zeit. Phys. C11, 293 (1982).

[37] J. C. Collins, Phys. Rev. D58, 094002 (1998), [hep-ph/9806259].

[38] G. Ingelman and P. E. Schlein, Phys. Lett. B152, 256 (1985).

[39] M. Gluck, E. Reya and A. Vogt, Z. Phys. C67, 433 (1995).

[40] M. Wusthoff, Phys. Rev. D56, 4311 (1997), [hep-ph/9702201].

[41] J. Bartels, J. R. Ellis, H. Kowalski and M. Wusthoff, Eur. Phys. J. C7, 443 (1999), [hep$\mathrm{ph} / 9803497]$.

[42] J. R. Forshaw, G. Kerley and G. Shaw, Phys. Rev. D60, 074012 (1999), [hep-ph/9903341].

[43] J. R. Forshaw, G. R. Kerley and G. Shaw, Nucl. Phys. A675, 80c (2000), [hep-ph/9910251].

[44] J. R. Forshaw, R. Sandapen and G. Shaw, Phys. Lett. B594, 283 (2004), [hep-ph/0404192].

[45] C. Marquet, arXiv:0706.2682 [hep-ph].

[46] G. Soyez, arXiv:0705.3672 [hep-ph].

[47] A. M. Stasto, K. Golec-Biernat and J. Kwiecinski, Phys. Rev. Lett. 86, 596 (2001), [hep$\mathrm{ph} / 0007192]$.

[48] K. Golec-Biernat and J. Kwiecinski, Phys. Rev. D55, 3209 (1997), [hep-ph/9607399]. 
[49] K. Golec-Biernat, J. Kwiecinski and A. Szczurek, Phys. Rev. D56, 3955 (1997), [hep$\mathrm{ph} / 9701254]$.

[50] H1, A. Aktas et al., Eur. Phys. J. C48, 749 (2006), [hep-ex/0606003].

[51] ZEUS, S. Chekanov et al., Eur. Phys. J. C38, 43 (2004), [hep-ex/0408009].

[52] ZEUS, S. Chekanov et al., Nucl. Phys. B713, 3 (2005), [hep-ex/0501060].

[53] A. D. Martin, W. J. Stirling and R. S. Thorne, Phys. Lett. B636, 259 (2006), [hepph/0603143].

[54] V. A. Khoze, A. D. Martin and M. G. Ryskin, hep-ph/0702213. 


\section{Diffractive structure function}

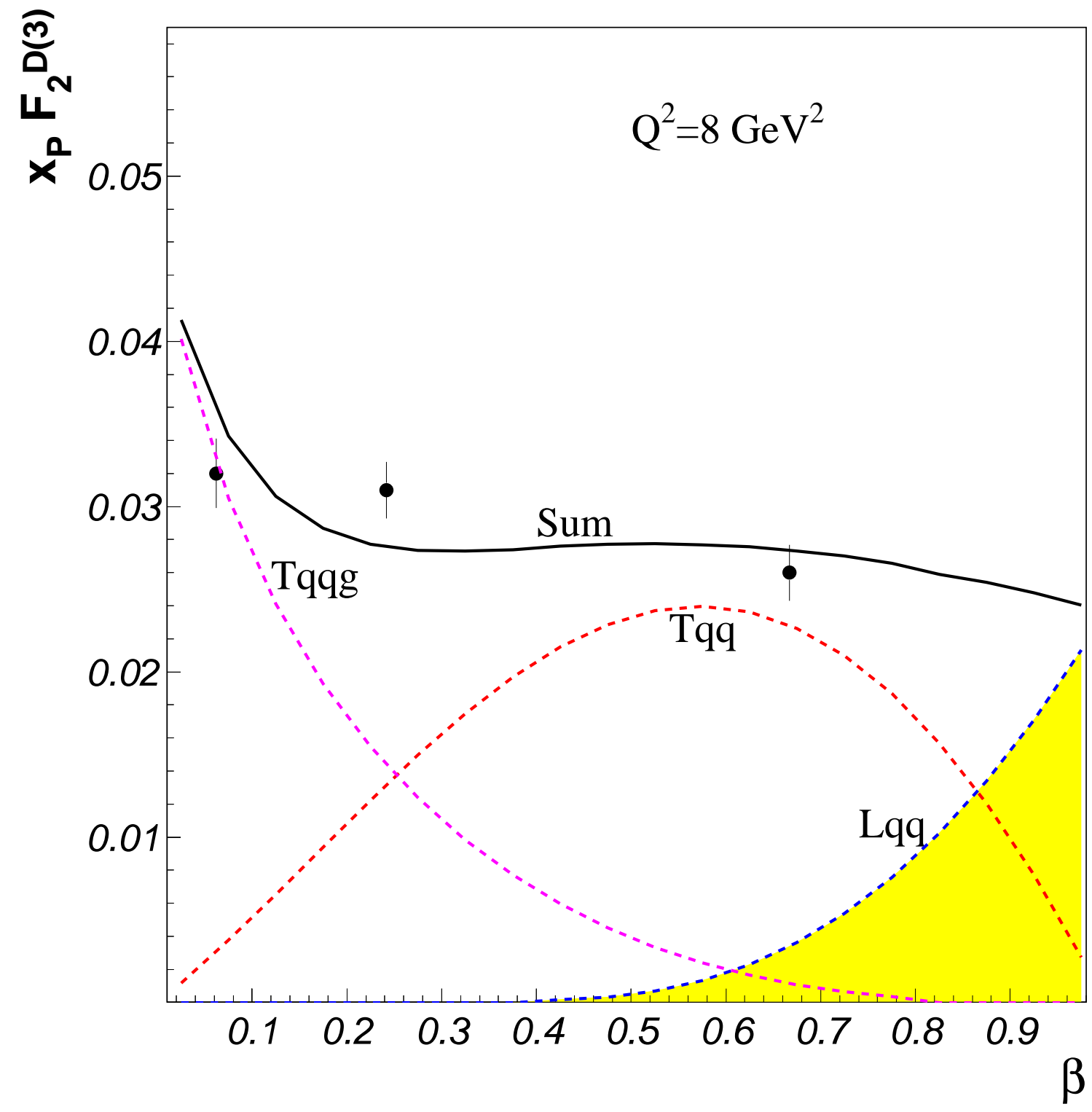

Figure 3: Three contributions to $F_{2}^{D}$ from: $q \bar{q}$ and $q \bar{q} g$ from transverse (T) and longitudinal (L) photons [19] for $x_{\mathbb{P}}=0.003$. The twist -4 contribution $L q \bar{q}$ is indicated by the yellow band. Old ZEUS data points are shown. 


\section{H1 DATA}

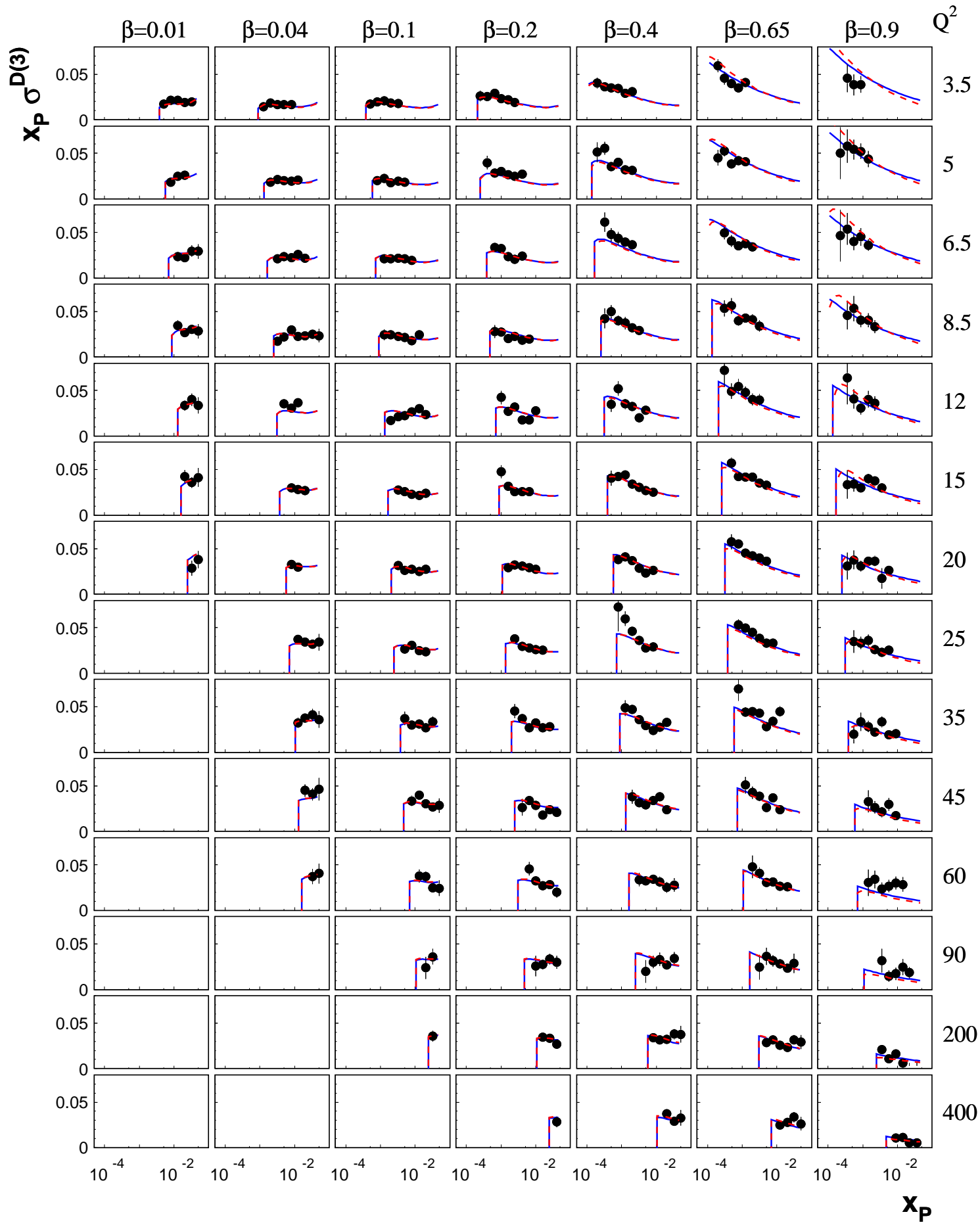

Figure 4: Reduced cross section $\sigma_{r}^{D(3)}$ for H1 data as a function of $x_{\mathbb{P}}$. Solid lines: twist-2 fit, dashed lines: twist- $-(2+4)$ fit. 
H1 DATA $(\beta=0.9)$
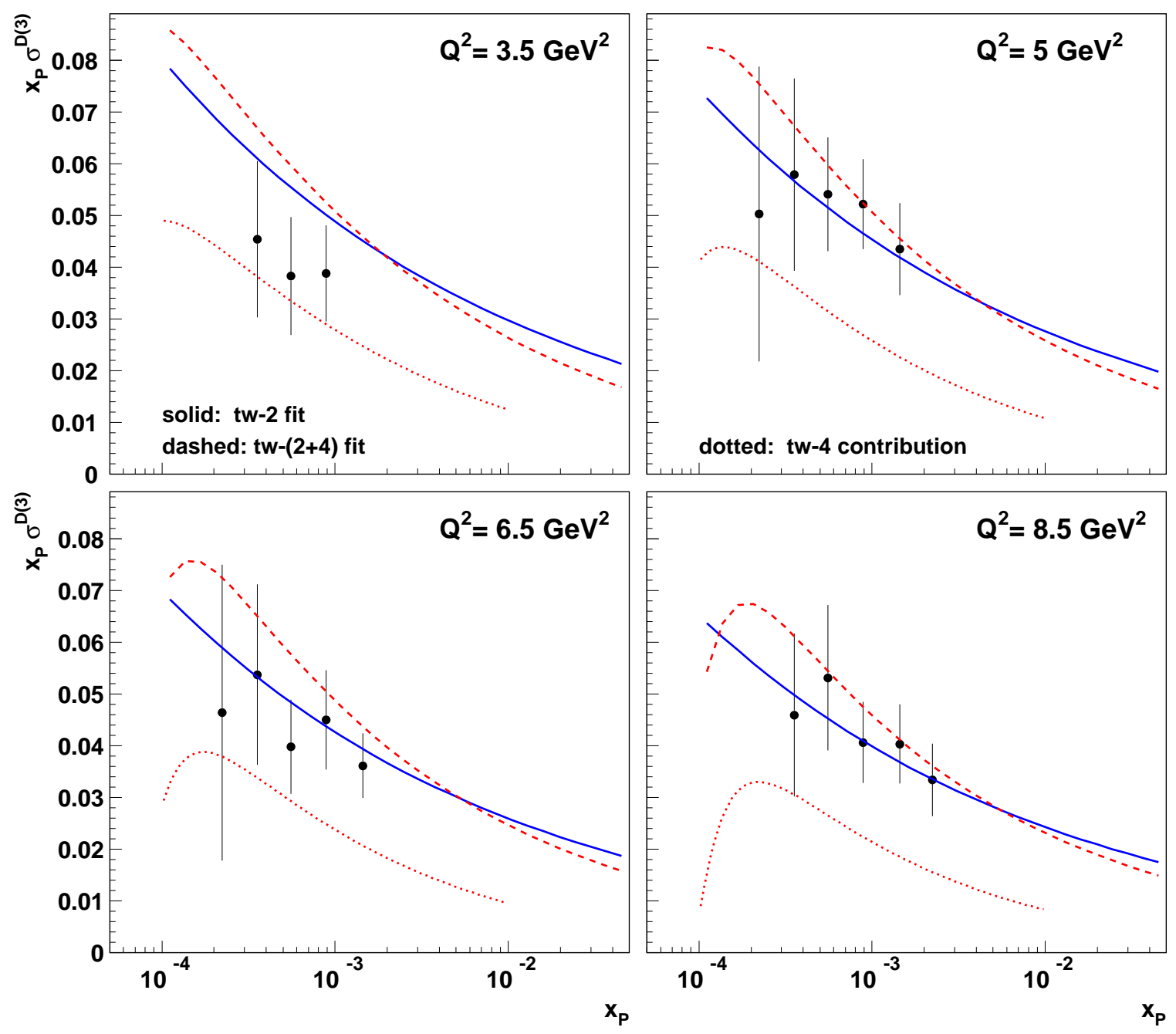

Figure 5: Reduced cross section $\sigma_{r}^{D(3)}$ for $\mathrm{H} 1$ data at $\beta=0.9$ for four values of $Q^{2}$ against fit curves. 


\section{DPD (H1)}
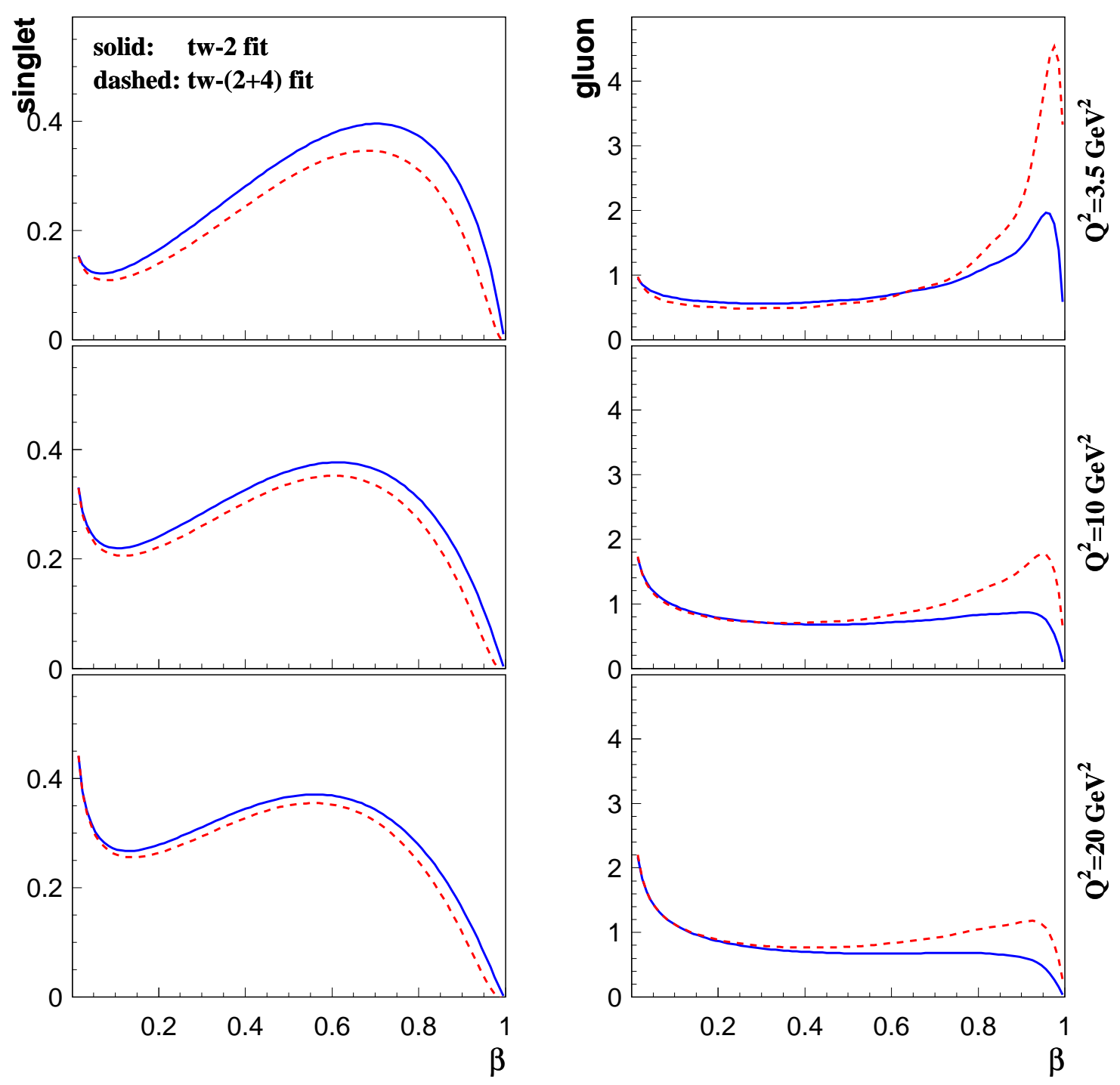

Figure 6: Pomeron parton distributions: singlet $\beta \Sigma_{\mathbb{P}}\left(\beta, Q^{2}\right)$ (left) and gluon $\beta g_{\mathbb{P}}\left(\beta, Q^{2}\right)$ (right) from $\mathrm{H} 1$ data. 


\section{DSF (H1)}
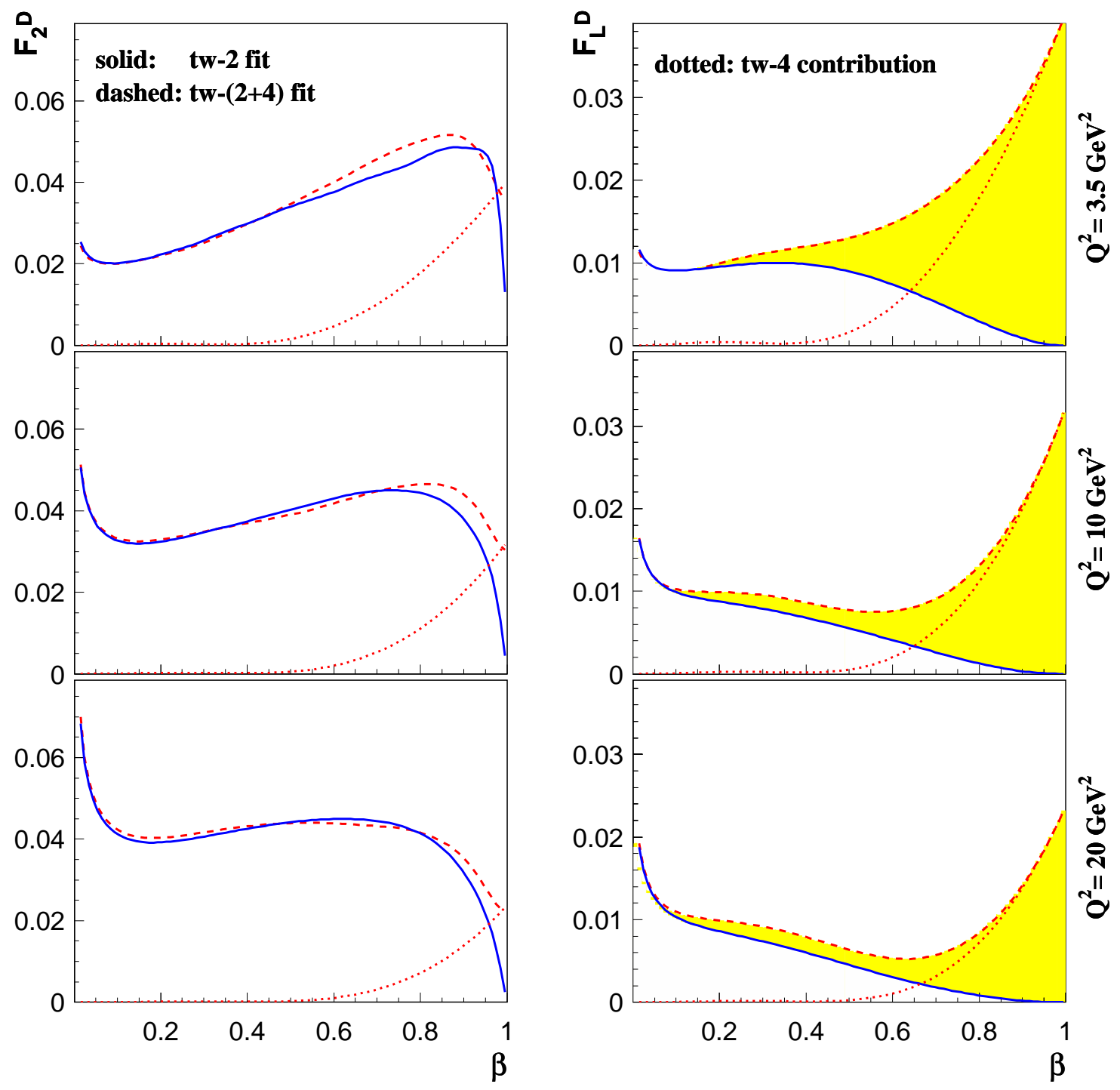

Figure 7: Diffractive structure functions $F_{2}^{D(3)}$ (left) and $F_{L}^{D(3)}$ (right) from fits to H1 data for $x_{\mathbb{P}}=10^{-3}$. The band shows the effect of twist-4 on the predictions for $F_{L}^{D(3)}$. 


\section{ZEUS DATA}

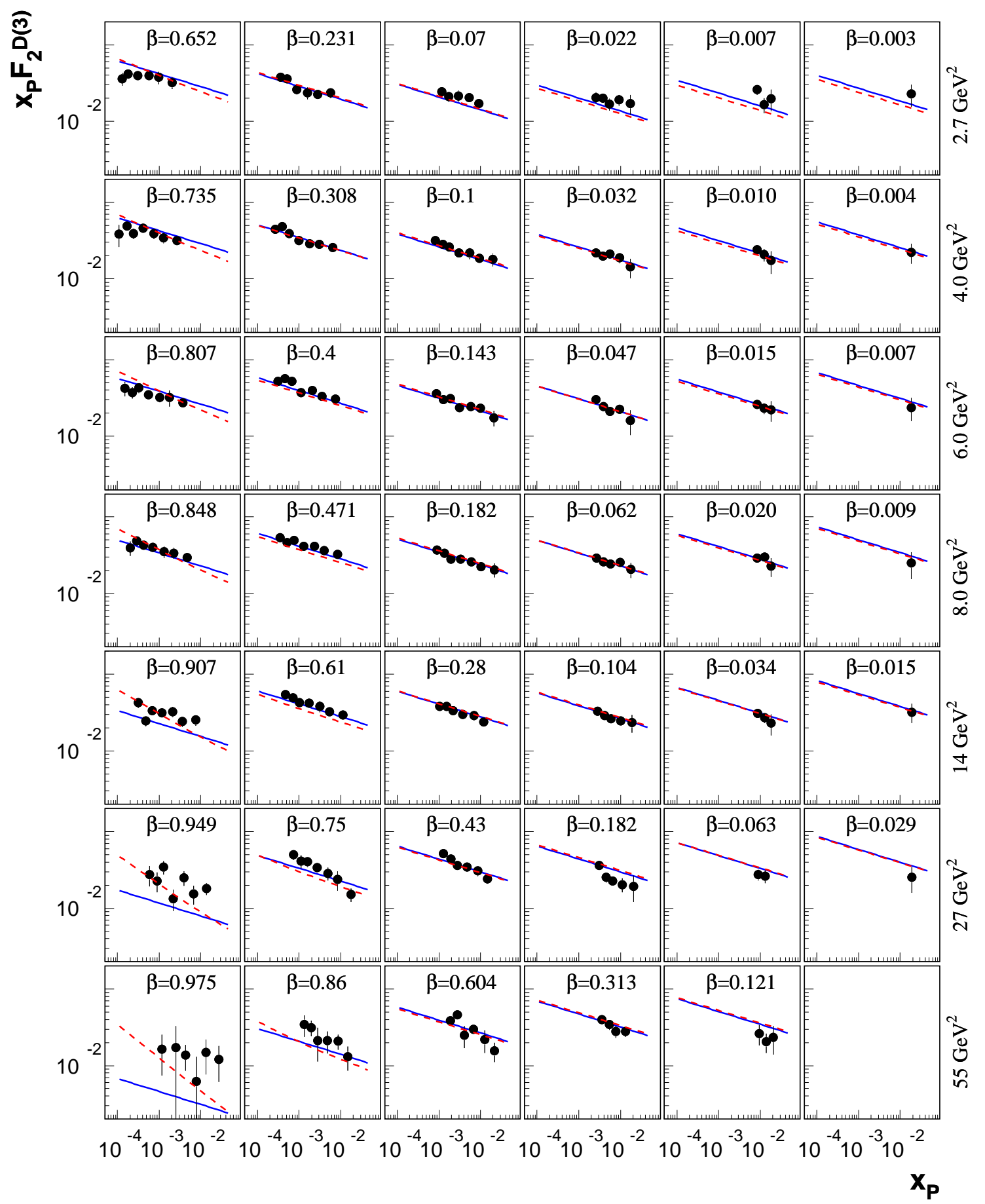

Figure 8: Diffractive structure function $F_{2}^{D(3)}$ as a function $x_{\mathbb{P}}$ for ZEUS data. Solid lines: twist-2 fit, dashed lines: twist-(2+4) fit. 


\section{ZEUS DATA}
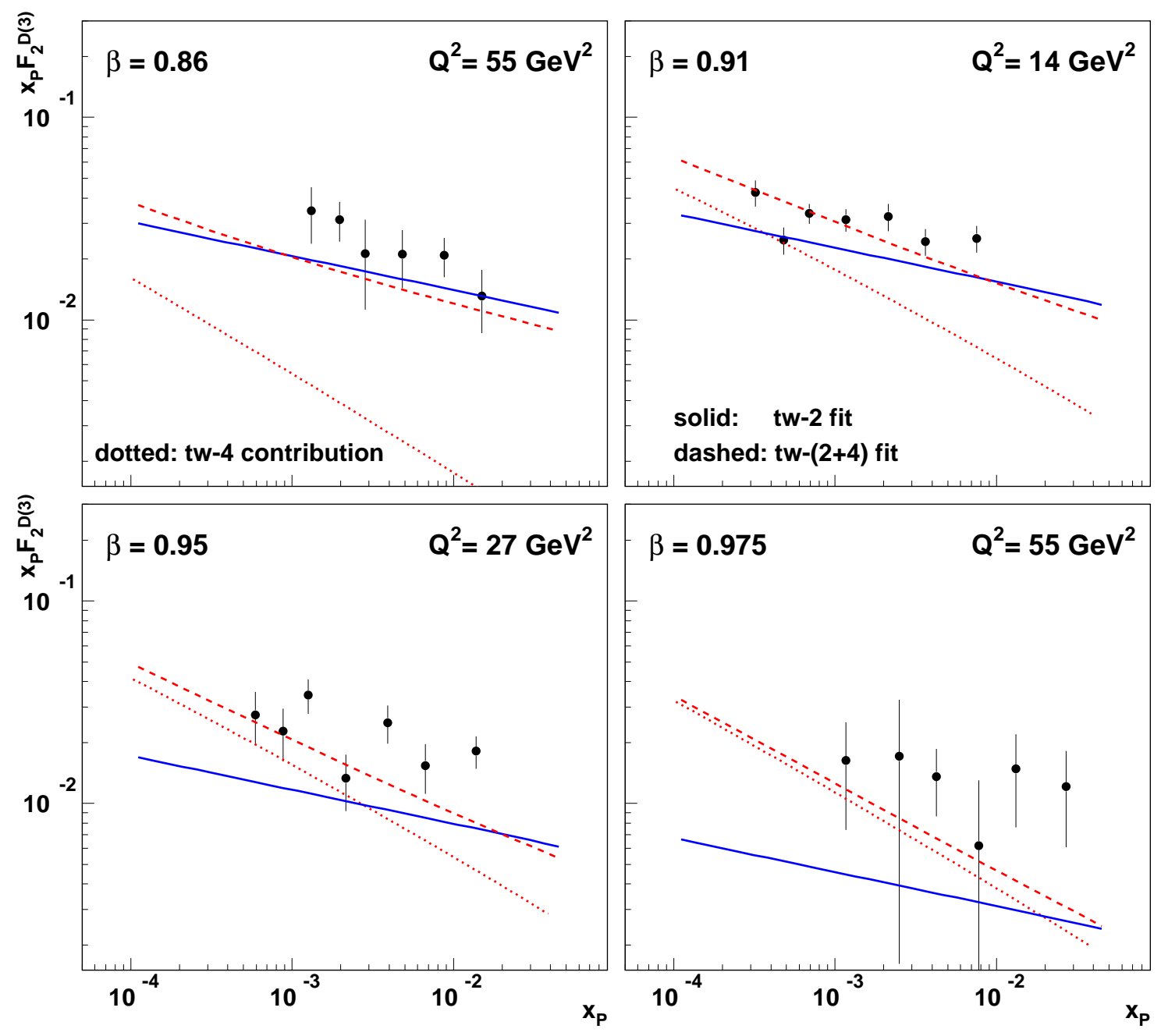

Figure 9: Diffractive structure function $F_{2}^{D(3)}$ for ZEUS data at large values of $\beta$ against fit curves. 


\section{DPD (ZEUS)}
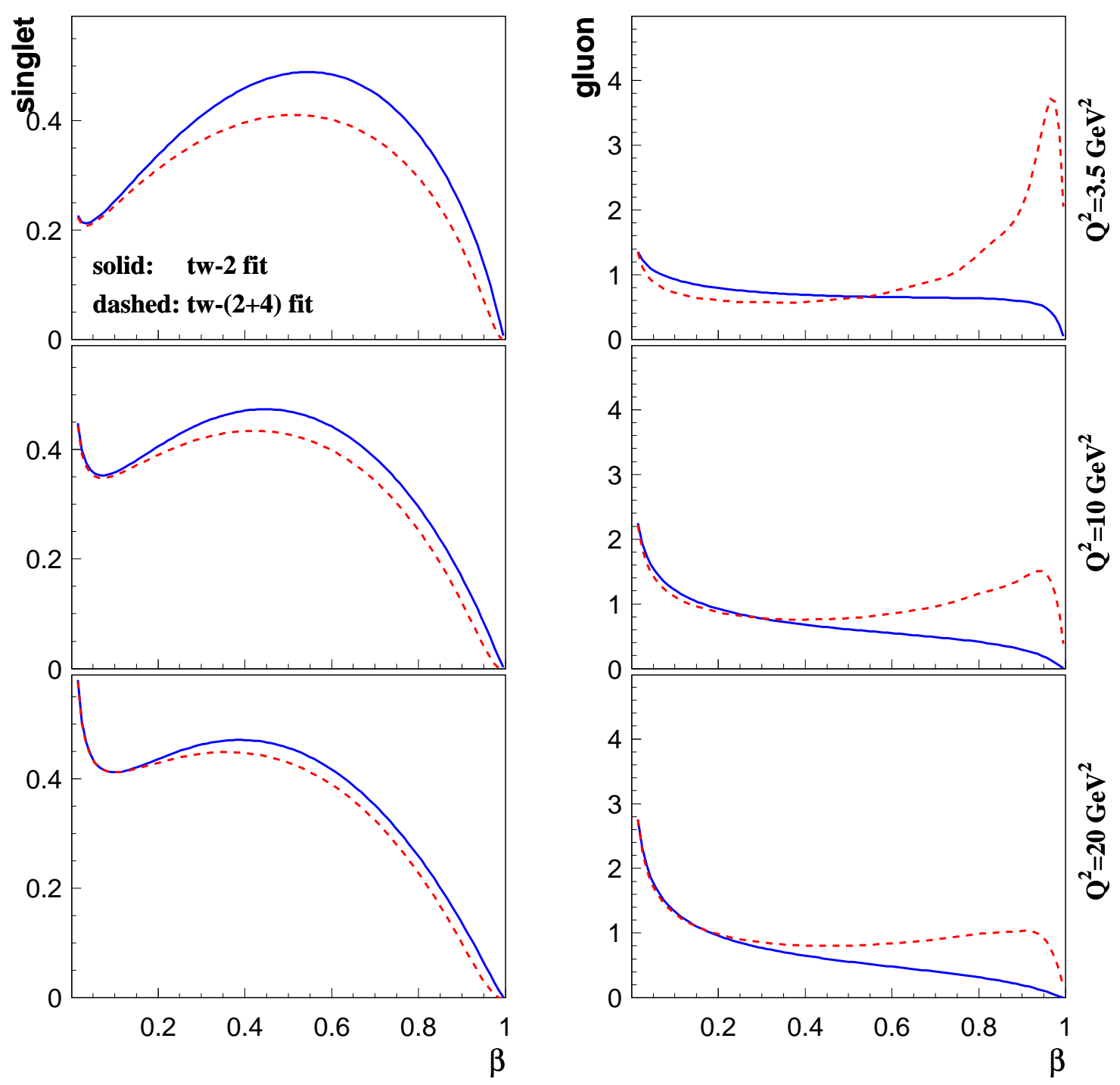

Figure 10: Pomeron parton distributions $\beta \Sigma_{\mathbb{P}}\left(\beta, Q^{2}\right)$ (left) and $\beta g_{\mathbb{P}}\left(\beta, Q^{2}\right)$ (right) from fits to ZEUS data. 


\section{DSF (ZEUS)}
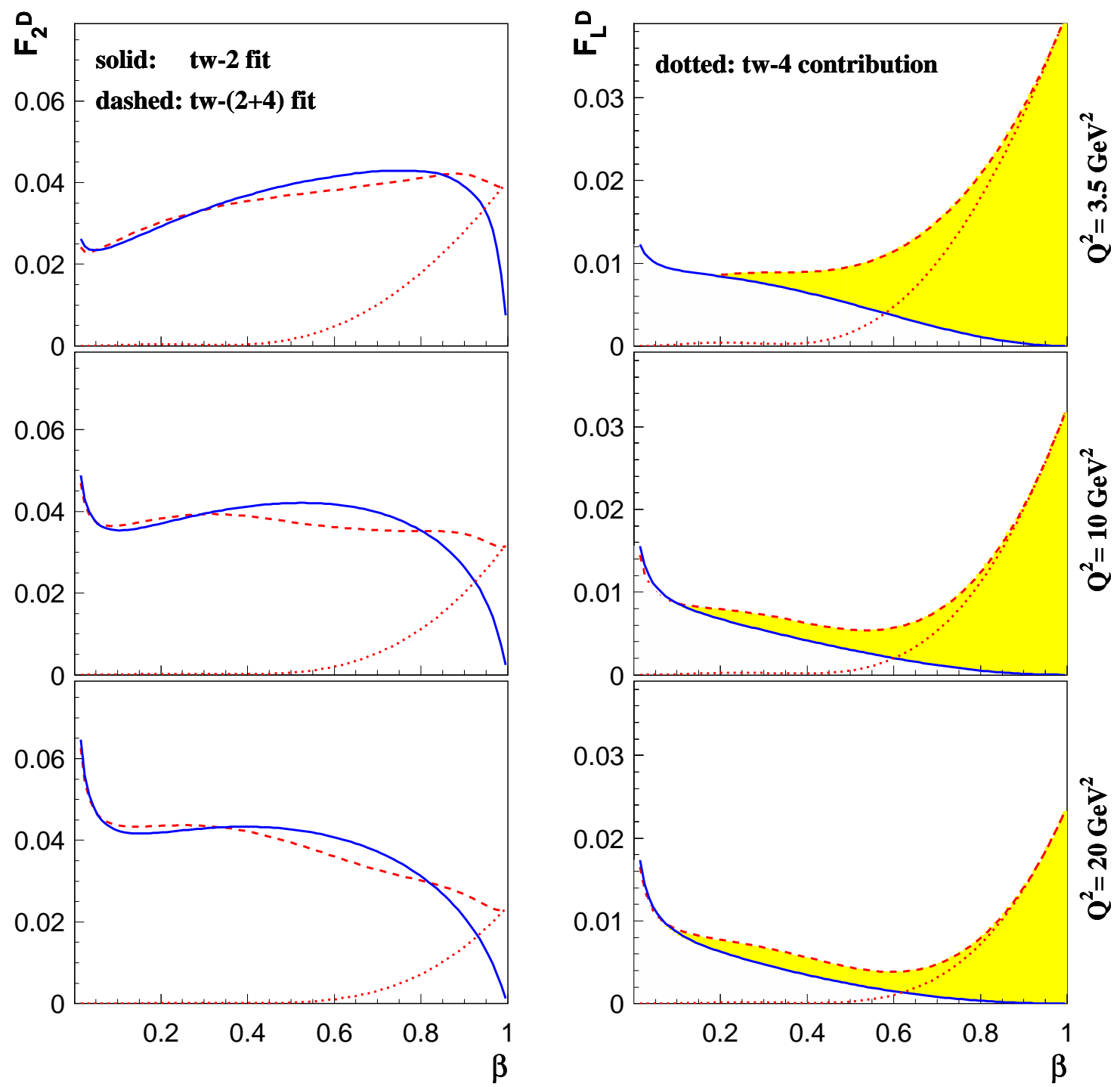

Figure 11: Diffractive structure functions $F_{2}^{D(3)}$ (left) and $F_{L}^{D(3)}$ (right) from fits to ZEUS data for $x_{\mathbb{P}}=10^{-3}$. The band shows the effect of twist-4 on the predictions for $F_{L}^{D(3)}$. 


\section{Diffractive $F_{L}$}

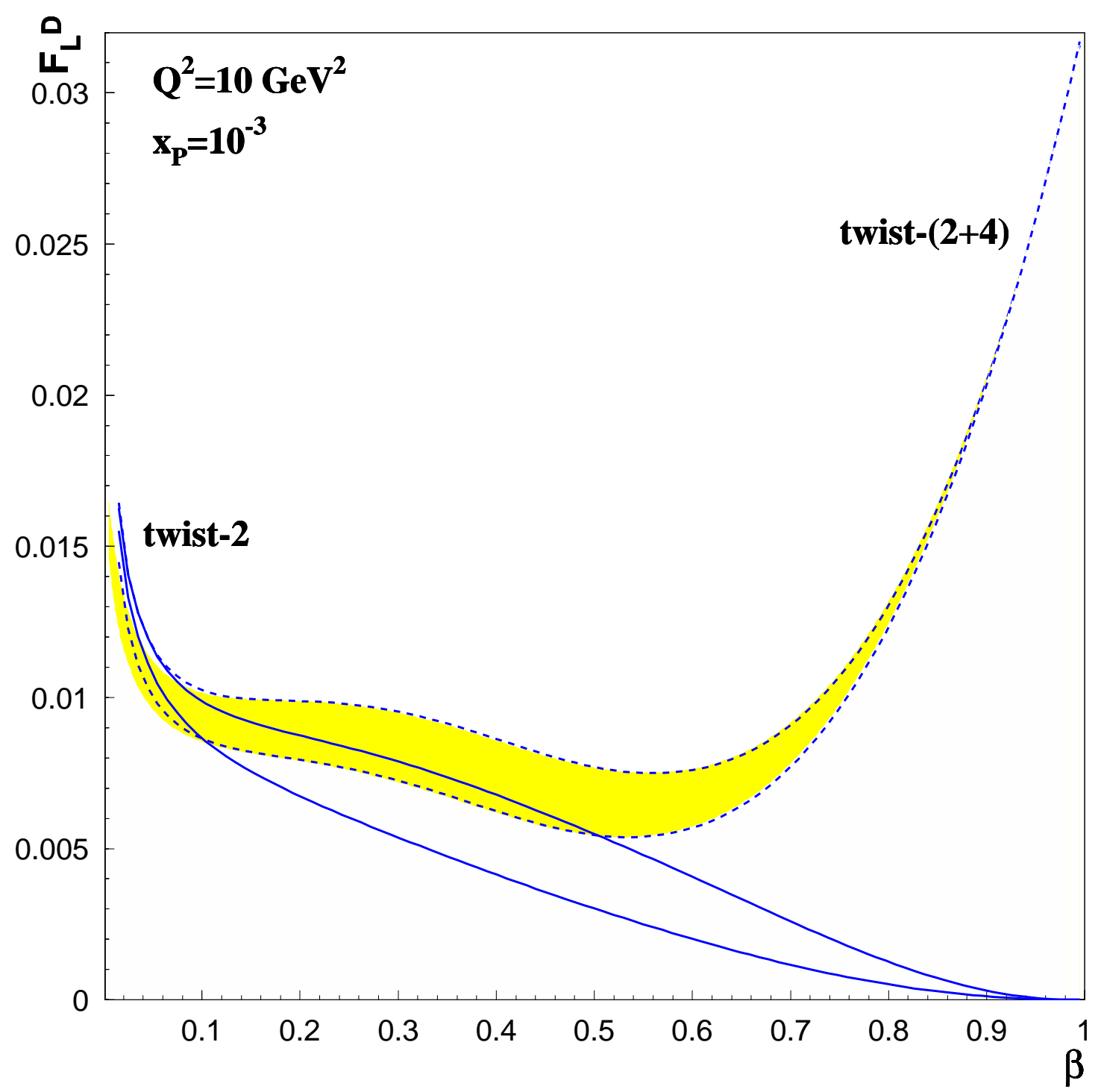

Figure 12: Predictions for $F_{L}^{D(3)}$ for $x_{\mathbb{P}}=10^{-3}$ and $Q^{2}=10 \mathrm{GeV}^{2}$ from the twist- $(2+4)$ fits to the H1 (upper dashed line) and ZEUS (lower dashed line) data. The solid lines show predictions from pure twist-2 fits to the H1 (upper) and ZEUS (lower) data. 